TÖFOLI, J.G.; DOMINGUES, R.J.; FERRERIRA, M.R.; GARCIA JÚNIOR, O. Ação de acibenzolar-s-methyl isolado e em mistura com fungicidas no controle da requeima da batata. Horticultura Brasileira, Brasília, v.23, n.3, p.749-753, jul-set 2005.

\title{
Ação de acibenzolar-s-methyl isolado e em mistura com fungicidas no controle da requeima da batata
}

\author{
Jesus G. Töfoli ${ }^{1}$; Ricardo J. Domingues ${ }^{1}$; Mariléia R. Ferreira ${ }^{1}$; Orlando Garcia Júnior ${ }^{2}$ \\ ${ }^{1}$ Instituto Biológico, Centro de P\&D de Sanidade Vegetal/Laboratório de Fungicisas e Filoprotetores, Av. Cons. Rodrigues Alves, 1252, \\ 04014-002 São Paulo-SP; ${ }^{2}$ Tecnocamp, Rua Salvador Rolim da Silva, 41, 18170-000 Piedade-SP; E-mail: tofoli@biologico.sp.gov.br
}

\section{RESUMO}

Visando avaliar o desempenho de acibenzolar-S-methyl (BTH) isolado e em mistura com os fungicidas mancozeb, chlorothalonil e metaxyl-M+chlorothalonil no controle da requeima da batata, foram realizados dois experimentos em cultivos comerciais nos municípios paulistas de Pilar do Sul (cv. Baraka) e Bragança Paulista (cv. Asterix), de maio a agosto e agosto a outubro de 2001, respectivamente. $\mathrm{O}$ delineamento experimental foi o de blocos ao acaso com 4 repetições, com cada parcela medindo $25 \mathrm{~m}^{2}$. Durante os experimentos foram realizadas um total de 10 pulverizações, a intervalos de 5 a 10 dias com pulverizador costal munido de barra de aplicação contendo 5 bicos TXKV26, pressão constante (4 bar) e volume médio de $800 \mathrm{~L} / \mathrm{ha}$. Avaliou-se a severidade nas folhas (0 a 100\%), nas hastes ( escala de notas de 1 a 5 ) e a produtividade comercial de tubérculos. O BTH apresentou efeitos significativos de controle quando utilizado isolado e em mistura com fungicidas de contato e sistêmico. Os maiores aumentos relativos de produtividade foram verificados para as misturas de BTH com mancozeb e chlorothalonil.

Palavras-chave: Phytophthora infestans, controle químico, indutor de resistência, BTH.

\section{ABSTRACT}

Action of acibenzolar-S-methyl, isolated and in a mixture with fungicides on potato late blight control

To evaluate the performance of acibenzolar-S-methyl (BTH), alone and mixed with mancozeb, chlorothalonil and metaxyl$\mathrm{M}+$ chlorothalonil, for the control of potato late blight, two experiments were carried out in commercial crops in the counties of Pilar do Sul (cv. Baraka) and Bragança Paulista (cv. Asterix), state of São Paulo, Brazil, from May to August and August to October 2001, respectively. The experimental design was of randomized blocks with four replications, with each plot measuring $25 \mathrm{~m}^{2}$. A total of 10 sprayings was done at 5- to 10-day intervals with a backpack sprayer using a 5-nozzle (TXKV26) spray wand, constant pressure of 4 bars (58 psi) and average volume of $800 \mathrm{~L} / \mathrm{ha}$. Evaluations were done in regard to the severity on the leaves ( 0 to $100 \%$ ), on the stems (score from 1 to 5) and the commercial yield of tubers. BTH presented expressive effects of control when used alone and in mixture with contact and systemic fungicides. Highest yield was verified for the mixture of BTH with mancozeb and chlorothalonil.

Keywords: Phytophthora infestans, chemical control, plant activator, BTH.

\section{(Recebido para publicação em 29 de junho de 2004 e aceito em 13 de maio de 2005)}

$\mathrm{A}$ lto potencial destrutivo, rápida disseminação e elevadas perdas econômicas, caracterizam a requeima causada por Phytophthora infestans como uma das mais importantes e destrutivas doenças da cultura da batata, em todo o mundo (ROWE, 1993; ZAMBOLIM et al., 2001). Cultivares suscetíveis ou com baixos níveis de resistência associados à temperaturas amenas e alta umidade, são fatores favoráveis à doença, que pode causar perda total da cultura em poucos dias (FRY, 1994).

Atualmente a utilização de fungicidas é medida necessária dentro de programas de manejo e sistemas de previsão da requeima, que visem elevados índices de produtividade e qualidade de tubérculos. O controle químico está fundamentado no uso preventivo de anti-oomicetos em mistura com produtos inespecíficos, bem como, o uso intercalado de fungicidas com diferentes mecanismos de ação. Trabalhos de pesquisa têm destacado o elevado potencial de controle de fungicidas como f a m ox a d o n e + c y m ox a n i 1 , dimetomorph + chlorothalonil, dimetomorph+mancozeb, metalaxyl$\mathrm{M}+\mathrm{ch} 1$ or ot h a 1 o n i 1 , propamocarb+chlorothalonil, cymoxanil+maneb+sulfato de zinco (CANTERI et al., 1993; TÖFOLI et al., 1999, TÖFOLI et al., 2001). Apesar de apresentar eficiência, o uso exclusivo de fungicidas pode ser considerado restrito quando são mencionadas questões como uso inadequado, impacto ambiental e o surgimento de raças resistentes de patógenos.

Pesquisas intensivas em busca de novos conceitos e alternativas para o controle de doenças têm levado ao desenvolvimento de substâncias capazes de induzirem o sistema de defesa da planta, tornando-a resistente à ação de patógenos sem a alteração de sua constituição genética básica (LEROUX, 1996; RYALS, 1996). A indução de resistência utiliza os mecanismos de defesa da própria planta para restringir o desenvolvimento do patógeno (SIGRIEST et al., 1997). Tal mecanismo de defesa pode ser induzido por microorganismos, extratos, ou ainda, pela aplicação de compostos químicos como o ácido salicílico e seus análogos. O benzotiadiazol acibenzolar-S-methyl (BTH), pertencente ao grupo quimico benzotiadiazole é considerado um ativador químico da resistência de plantas a doenças. Após sua aplicação o BTH é absorvido e translocado pela planta, gerando um sinal sistêmico que desencadeia a expressão dos genes de defesa, ou seja o fenômeno da resistência sistêmica adquirida (SAR) (STICHER et al., 1997; WEI et al., 1991; WHITE et al., 1979; YAMAGUCHI, 1998). A 
aquisição de resistência através de BTH deve-se principalmente ao acúmulo nas células de PR-proteínas como as proteinases, quitinases e peroxidases. Tais proteínas apresentam a capacidade de degradar as paredes celulares de fungos e bactérias, impedindo o processo infeccioso (MADAMANCHI et al., 1991).

Estudos sobre o potencial de controle de acibenzolar-S-methyl têm sido realizados com sucesso em diversos patossistemas como oídio em trigo (GÖRLACH et al., 1996); míldio em tabaco (FRIEDRICH et al., 1996) míldio em Arabidopsis (LAWTON et al., 1996); ferrugem do feijão-vagem (SIGRIEST et al., 1997); pinta preta do tomateiro (TÖFOLI, 2002) e cancro da haste em melão rendilhado (RIZZO et al., 2003). Baixa toxicidade inerente, modo de ação inespecífico e baixo risco de resistência tornam o BTH uma alternativa a ser considerada em programas de manejo de doenças.

O objetivo do presente trabalho foi avaliar o potencial de integração entre acibenzolar-S-methyl e fungicidas de contato e sistêmicos, no controle da requeima da batateira.

\section{MATERIAL E MÉTODOS}

Em condições de plantio comercial de batata, foram realizados dois experimentos nos municípios paulistas de Pilar do Sul (maio a agosto, 2001, experimento 1) e Bragança Paulista (agosto a outubro, 2001, experimento 2) utilizando as cultivares Baraka e Asterix, respectivamente. O delineamento experimental utilizado foi o de blocos ao acaso com 13 tratamentos e 4 repetições, sendo cada parcela composta por $25 \mathrm{~m}^{2}$. Os tratamentos avaliados e as respectivas doses (g ou $\mathrm{kg}$ de p.c./ ha) foram: 1) Chlorothalonil=1,5; 2) Chlorothalonil (2/3) (produto com redução de $1 / 3$ da dose $)=1,0 ; 3$ ) Mancozeb=3,0; 4) Mancozeb (2/3) (produto com redução de $1 / 3$ da dose) $=2,0 ; 5$ ) BTH (acibenzolar-S-methyl) $=25$; 6) BTH + Chlorothalonil $=25+1,5 ; 7) \mathrm{BTH}+$ Chlorothalonil (2/3) ${ }^{1}$ (produto com redução de $1 / 3$ da dose) $=25+1,0 ; 8) \mathrm{BTH}+$ Mancozeb $=\mathrm{BTH}+$ Mancozeb $=25+3,0$; 9) $\mathrm{BTH}+$ Mancozeb (2/3) ${ }^{1}$ (produto com redução de $1 / 3$ da dose $)=25+2,0 ; 10$ ) Metalaxyl-M+Chlorothalonil/
Chlorothalonil $^{2}$ (cinco aplicações do primeiro produto seguidas de cinco do segundo) $=1,5$; 11) BTH+Metalaxyl$\mathrm{M}+$ Chlorothalonil/Chlorothalonil $^{2}$ (cinco aplicações do primeiro produto seguidas de cinco do segundo) $=25+1,5 ; 12$ ) Cymoxanil+Maneb+ZnSO Chlorothalonil $^{2}$ (cinco aplicaçõ̃es do primeiro produto seguidas de cinco do segundo) $=2,0 ; 13$ ) Testemunha.

As aplicações tiveram inicio aos 35 dias após a emergência (DAE) no experimento 1 e aos 30 DAE no experimento 2 . Foram realizadas preventivamente, em intervalos de 5 a 10 dias, utilizando-se um pulverizador costal munido de barra de aplicação e pressão constante de 4 bar $\left(\mathrm{CO}_{2}\right)$. A barra de aplicação continha 5 bicos cônicos do tipo TXKV26, espaçados de 0,5 m, sendo a distância entre a barra e alvo durante a aplicação de aproximadamente $0,5 \mathrm{~m}$. $\mathrm{O}$ volume de aplicação variou de 600 a 1000 L/ha, em função do desenvolvimento da cultura, perfazendo um total de 10 pulverizações por tratamento.

No decorrer dos experimentos foram adotados todos os tratos culturais recomendados para o cultivo da batata.

Avaliou-se a severidade da doença, através da análise visual da porcentagem de área foliar afetada pela doença (0 a 100\%), em $10 \mathrm{~m}^{2}$ centrais das parcelas. No experimento 1, a avaliação foi realizada aos 82 DAE e no experimento 2 aos 78 DAE. Foi avaliada também a ocorrência de sintomas na haste, com o uso de uma escala de notas de 1 a 5 onde, $1=$ ausência de sintomas; 2=lesões menores que $0,5 \mathrm{~cm} ; 3=$ lesões entre 0,5 e $3 \mathrm{~cm} ; 4=$ lesões maiores que $3 \mathrm{~cm}$; 5=lesões que envolviam toda a haste e morte de ponteiro. Esta coincidiu com a avaliação da porcentagem de área foliar afetada de cada experimento. Por fim, avaliou-se a produtividade (kg de tubérculos) em $10 \mathrm{~m}^{2}$ centrais das parcelas e calculou-se o aumento relativo desta pela adição de BTH a fungicidas.

Os dados foram analisados estatisticamente pela análise da variância, aplicando-se o teste de Tukey a 5\% de probabilidade para comparação das médias. Os dados de porcentagem de área foliar foram previamente transformados em arc sen raiz de $\mathrm{x} / 100$.

\section{RESULTADOS E DISCUSSÃO}

A suscetibilidade das cultivares utilizadas no presente estudo, associada à condições favoráveis permitiram que a requeima alcançasse elevados níveis de severidade e causasse significativa redução da produtividade nas parcelas testemunhas. Todos os tratamentos diferiram significativamente da testemunha quando avaliada a severidade da doença em folhas (Tabelas 1 e 2).

Em ambos os experimentos a mistura $\mathrm{BTH}+$ metalaxyl-M+chlorothalonil seguida de metalaxyl-M+chlorothalonil e cymoxanil+maneb+sulfato de zinco proporcionaram os melhores níveis de controle da requeima, sendo superiores aos demais tratamentos. Chlorothalonil e mancozeb promoveram níveis intermediários de controle, sendo geralmente semelhantes entre si. A redução de 1/ 3 na dose de chlorothalonil e mancozeb em relação à dose completa somente afetou o potencial de controle destes fungicidas no experimento 2 .

A aplicação de BTH proporcionou os menores níveis de controle da doença, sendo semelhante ao mancozeb em dose reduzida e inferior aos demais tratamentos. Em ambos os experimentos a adição de BTH a metalaxylM+chlorothalonil, chlorothalonil e mancozeb promoveu incremento significativo no controle da requeima, em relação ao uso destes fungicidas isoladamente. Os tratamentos mancozeb e chlorothalonil em mistura com BTH, independentemente de dose, não diferiram estatisticamente entre si.

Com relação à ocorrência de requeima em hastes, no experimento 1 , somente as parcelas tratadas com dose reduzida de chlorothalonil e mancozeb apresentaram traços da doença, todavia em níveis inferiores à testemunha. No experimento 2, verificou-se a mesma tendência de controle verificada para o critério porcentagem de área foliar afetada. A adição de BTH promoveu incremento significativo do controle da requeima em haste para os tratamentos com mancozeb e chlorothalonil independente da redução de dose. De maneira geral, as doses de chlorothalonil e mancozeb não diferiram entre si com 
Tabela 1. Severidade da requeima da batata em folhas, hastes e produtividade de plantas de batata, cv. Baraka, tratadas com fungicidas e acibenzolar-S-methyl, Pilar do Sul (SP), 2001.

\begin{tabular}{|c|c|c|c|c|}
\hline \multirow[b]{2}{*}{ Tratamentos } & \multicolumn{2}{|c|}{ Severidade } & \multicolumn{2}{|c|}{ Produtividade } \\
\hline & $\begin{array}{l}\% \text { de área foliar } \\
\text { afetada ( } 82 \text { DAE) }\end{array}$ & Haste (82 DAE) ${ }^{1}$ & $\left(\mathrm{Kg} / 10 \mathrm{~m}^{2}\right)$ & $\begin{array}{c}\text { Aumento relativo pela } \\
\text { adição de BTH (\%) }\end{array}$ \\
\hline Chlorothalonil & $44,3^{2} \mathrm{de}^{3}$ & 1,00 & 12,0 bcd 2 & \\
\hline BTH+ Chlorothalonil & $36,5 \mathrm{f}$ & 1,00 & $15,0 \mathrm{bc}$ & 25,0 \\
\hline Chlorothalonil $(2 / 3)$ & $52,7 \mathrm{~cd}$ & 1,18 & $9,8 \mathrm{cde}$ & \\
\hline BTH+ Chlorothalonil $(2 / 3)$ & 39,8 ef & 1,00 & $12,5 \mathrm{bcd}$ & 28,2 \\
\hline Mancozeb & $51,3 \mathrm{~cd}$ & 1,00 & $11,8 \mathrm{bcd}$ & \\
\hline BTH+ Mancozeb & 40,0 ef & 1,00 & $16,8 \mathrm{~b}$ & 42,6 \\
\hline Mancozeb (2/3) & 58,7 bc & 1,25 & 9,0 cde & \\
\hline BTH+ Mancozeb (2/3) & 44,2 def & 1,00 & $15,0 \mathrm{bc}$ & 66,7 \\
\hline BTH (acibenzolar-S-methyl) & $65,1 \mathrm{~b}$ & 1,00 & $7,3 \mathrm{de}$ & \\
\hline Metalaxyl-M+ Chlorothalonil & $8,3 \mathrm{~g}$ & 1,00 & 24,3 a & \\
\hline BTH+ Metalaxyl-M+ Chlorothalonil & $4,0 \mathrm{~h}$ & 1,00 & 28,8 a & 18,6 \\
\hline Cymoxanil+ Maneb+ $\mathrm{ZnSO}_{4}$ & $11,8 \mathrm{~g}$ & 1,00 & 25,5 a & \\
\hline Testemunha & 82,9 a & 3,25 & 6,8 e & \\
\hline CV (\%) & 10,49 & & 15,39 & \\
\hline
\end{tabular}

${ }^{1}$ DAE - dias após a emergência; ${ }^{2}$ Médias originais: Para analise estatística os dados foram transformados arc sen raiz de x/100. ${ }^{3}$ Médias seguidas de mesma letra não diferem entre si pelo teste de Tukey a $5 \%$ de probabilidade

Tabela 2. Severidade da requeima da batata em folhas, hastes e produtividade de plantas de batata, cv. Asterix, tratadas com fungicidas e acibenzolar-S-methyl, Bragança Paulista (SP), 2001.

\begin{tabular}{|c|c|c|c|c|}
\hline \multirow[b]{2}{*}{ Tratamentos } & \multicolumn{2}{|c|}{ Severidade } & \multicolumn{2}{|c|}{ Produtividade } \\
\hline & $\begin{array}{l}\% \text { de área foliar } \\
\text { afetada (78 DAE) }\end{array}$ & Haste $(78 \mathrm{DAE})^{1}$ & $\left(\mathrm{Kg} / 10 \mathrm{~m}^{2}\right)$ & $\begin{array}{c}\text { Aumento relativo pela } \\
\text { adição de BTH (\%) }\end{array}$ \\
\hline Chlorothalonil & $52,3^{2} \mathrm{de}^{3}$ & $2,8 \quad b$ & $11,6 \mathrm{~cd}$ & \\
\hline BTH+ Chlorothalonil & $37,5 \mathrm{f}$ & $1,9 \mathrm{c}$ & $15,3 \mathrm{~b}$ & 31,5 \\
\hline Chlorothalonil $(2 / 3)$ & $64,6 \mathrm{c}$ & $2,9 \mathrm{~b}$ & $10,3 \mathrm{~cd}$ & \\
\hline BTH+ Chlorothalonil $(2 / 3)$ & 47,5 def & $1,4 \mathrm{~cd}$ & $15,8 \mathrm{~b}$ & 43,9 \\
\hline Mancozeb & $58,8 \mathrm{~cd}$ & $1,9 \mathrm{c}$ & $12,1 \mathrm{~cd}$ & \\
\hline BTH+ Mancozeb & $39,9 \mathrm{f}$ & $1,2 \mathrm{~d}$ & $16,3 \mathrm{~b}$ & 34,3 \\
\hline Mancozeb (2/3) & $65,6 \mathrm{~b}$ & $2,9 \mathrm{~b}$ & $11,3 \mathrm{~cd}$ & \\
\hline BTH+ Mancozeb (2/3) & 45,0 ef & $1,6 \mathrm{c}$ & $16,5 \mathrm{~b}$ & 46,7 \\
\hline BTH (acibenzolar-S-methyl) & $75,0 \quad b$ & $1,8 \quad \mathrm{c}$ & $8,8 \mathrm{~d}$ & \\
\hline Metalaxyl-M+ Chlorothalonil & $9,4 \mathrm{~g}$ & $1,0 \mathrm{~d}$ & 27,5 a & \\
\hline BTH+ Metalaxyl-M+ Chlorothalonil & $3,2 \mathrm{~h}$ & $1,0 \mathrm{~d}$ & 30,0 a & 9,1 \\
\hline Cymoxanil+ Maneb+ $\mathrm{ZnSO}_{4}$ & $12,1 \mathrm{~g}$ & $1,0 \mathrm{~d}$ & 26,3 a & \\
\hline Testemunha & $100,0 \quad a$ & $4,2 \quad a$ & $5,2 \mathrm{e}$ & \\
\hline CV (\%) & 6,13 & 12,54 & & \\
\hline
\end{tabular}

${ }^{1}$ DAE - dias após a emergência; ${ }^{2}$ Médias originais: Para analise estatística os dados foram transformados em arc sen raiz de x/100.

${ }^{3}$ Médias seguidas de mesma letra não diferem entre si pelo teste de Tukey s $5 \%$ de probabilidade.

exceção de chlorothalonil e mancozeb em dose reduzida no experimento 2 .

Os maiores níveis de produção, em ambos os experimentos, foram obtidos nos tratamentos BTH+metalaxyl$\mathrm{M}+$ chlorothalonil, metalaxylM+chlorothalonil, e cymoxanil+ maneb+sulfato de zinco que apresentaram comportamento superior aos demais tratamentos. Com exceção de BTH, mancozeb e chlorothalonil nas doses reduzidas, no experimento 1 , os demais tratamentos proporcionaram produção superior à testemunha.

Maior produção pela adição de BTH a fungicidas foi observada nas parcelas tratadas com mancozeb e chlorothalonil, independente de dose, no experimento 2. Apesar da mistura metalaxyl$\mathrm{M}+$ chlorothalonil + BTH proporcionar aumento do controle da requeima, em relação ao seu uso isolado e ao cymoxanil+maneb+sulfato de zinco, não verificou-se diferença significativa de produção entre estes.

A adição do indutor de resistência BTH em misturas com chlorothalonil e mancozeb proporcionou aumento significativo do controle da requeima e maior produtividade de tubérculos (Tabelas 1 
e 2). Para a cultivar Baraka verificou-se aumentos relativos de 25,0 a 28,2 e 42,6 a $66,7 \%$ nos tratamentos com chlorothalonil e mancozeb nas doses inteira e reduzida, respectivamente. Semelhantemente, para a cultivar Asterix, observou-se incrementos da ordem de 31,5 a $43,9 \%$ para chlorothalonil e 34,3 a $46,7 \%$ para mancozeb. Quanto a metalaxyl$\mathrm{M}+$ chlorothalonil, o incremento na produção foi menor em ambas as cultivares, alcançando níveis de 18,6 e 9,1\%, respectivamente.

Decréscimos no controle da requeima e da produtividade pela redução de dose de mancozeb e chlorothalonil foram observados somente no experimento 2. Neste, a adição de BTH a estes fungicidas promoveu resultados semelhantes aos obtidos com dose inteira.

Resultados sobre o aumento do controle e maiores níveis de produtividade pelo uso de BTH associado a fungicidas foram verificados para alguns patossistemas. Yamaguchi (1998) destaca que BTH aplicado em trigo isolado ou em mistura com cyprodinil e fenpropidin apresentaram aumento do controle do oídio e ferrugem, bem como incrementos na produção de 9; 13 e $17 \%$, respectivamente. Em experimentos onde se aplicaram fungicidas triazóis em esquema semelhante de tratamentos, verificou-se acréscimos de $40 \%$ no rendimento. Em estudos realizados por Silva e tal (2003) foi verificado que BTH em mistura com mancozeb proporcionou as maiores reduções de severidade de Xanthomonas vesicatoria, Oidium lycopersici e Septoria lycopersici em tomateiro. Semelhantemente, Rizzo et al. (2003) verificaram elevado potencial de controle da combinação BTH e difenoconazole no controle do cancro da haste do melão rendilhado. O efeito positivo da adição de BTH em programas de aplicação de fungicidas em tomateiro foi observado por Castro et al. (2000; 2001), que constataram redução significativa da ocorrência de requeima, pinta preta, septoriose e mancha bacteriana, bem como incrementos na produção e qualidade de frutos.

A ação sinergística da mistura de metalaxyl-M com BTH verificada neste trabalho também foi observada por
Molina et al. (1998) no controle de Phytophthora parasitica em Arabidopsis sp. Navia et al. (2001) destacam BTH como promissor no controle da requeima da batata dentro de estratégias de controle com fungicidas.

As adições de BTH a mancozeb, chlorothalonil e metalaxyl$\mathrm{M}+\mathrm{chlorothalonil} \mathrm{proporcionaram} \mathrm{me-}$ lhor controle da requeima da batata quando comparada ao uso isolado. Por outro lado, os maiores aumentos relativos de produtividade foram observados para as misturas com mancozeb e chlorothalonil. No presente estudo a adição de BTH a metalaxy$\mathrm{Ml}+$ chlorothalonil promoveu acréscimos não significativos a produção em relação ao uso isolado. Este fato devese provavelmente a uma menor contribuição da indução de resistência no âmbito geral do controle da requeima, visto a ação sistêmica do metalaxyl-M.

No presente estudo, os resultados obtidos demonstram o potencial do uso de BTH em mistura com metalaxyl$\mathrm{M}+$ chlorothalonil, chlorothalonil e mancozeb como uma alternativa adicional no manejo da requeima da batata. Tal fato confirma a viabilidade de integrar-se diferentes princípios e estratégias de controle, bem como abre perspectivas para a proteção integrada de plantas contra fitopatógenos.

\section{LITERATURA CITADA}

CANTERI, M.G., FURIATTI, R.S., PERINO, M.A. Efeito de fungicidas aplicados isoladamente ou em mistura no controle da requeima (Phytophthora infestans) da batata. Summa Phytopathologica, Jaboticabal, v.19, n. 2, p.195198, 1993.

CASTRO, R.M., VIEIRA, M., SCANAVACHI, E., GUICHERIT, E. Redução na severidade de doenças e incremento da produção e qualidade dos frutos de tomate estaqueado em áreas comerciais através da aplicação do ativador de plantas acibenzolar-methyl. Fitopatologia Brasileira, Brasília, v.25, suplemento, p.457, 2000.

CASTRO, R.M., VIEIRA, M., SCANAVACHI, V., AZEVEDO, L.A. Efeito do ativador de plantas acibenzolar-methyl na proteção contra doenças, incremento de produção e qualidade de frutos em tomate estaqueado. Fitopatologia Brasileira, Brasília, v.26, suplemento, 492. 2001.

FRIEDRICH, L., LAWTON, K., RUESS, W., MASNER, P., SPECKER, N., RELLA, M., GUT, M., MEIER, B., DINCHER, S., STAUB, T., UKNES, S., METRAUX, J.P., KESSMANN, H., RYALS, J.A. Benzothiadiazole derivate induces sistemic acquired resistance in tobacco. Plant Journal, v.10, n.1, p.61-70, 1996.
FRY, W.E. Role of early and late blight suppression in potato. In: ZENDER, G.W., POWELSON, M.L., JANSSON, R.K., RAMAN, K.V. Advances in potato pest - Biology and management. S Paul, American Phytopathological Society, 1994. p.166-177.

GÖRLACH, J., VOLRATH, S., KNAUFBEITER, G., HENGY, G., BECKHOVE, U., KOGEL, K.G., OOTENDORP, M., STAUB, T., WARDE, E., KESSMANN, J., RYALS, J. Benzothiadiazole, a novel class of inducers of sistemic acquired resistance, activates gene expression and disease in wheat. The Plant Cell, Rockville, v.8, n.3, p.629-643, 1996.

LAWTON, K.A., FRIEDRICH, L., HUNT, M., WEYMAN, K., DELANEY, T., KESSMANN, H., STAUB, T., RYALS, J. Benzothiadiazole induces disease resistance in Arabidopsis by activation of the sistemic acquired resistance signal transduction pathway. Plant Journal, v.10, n.1, p.71-82, 1996. LEROUX, P. Recent developments in the mode action of fungicides. Pesticide Science, v.47, n.3, p.191-197, 1996.

MADAMANCHI, N.R., KUC, J. Induced sistemic resistance in plants. In: COLE, G.T.; $\mathrm{HOCH}, \mathrm{H}$. (Eds) The fungal spore and disease initiation in plants. New York: Plenum Press, 1991. p.347-362. NAVIA, O., TRUJILLO, A., GANDARILLAS, A., GABRIEL, J., FERNÁNDEZ NORTHCOTE, E.N. Utilización de un activador de resistencia y funguicidas en estratégias de control químico del tizón de la papa en zonas muy tizoneras. Fitopatologia Brasileira, Brasília, v.26, 2001. Suplemento. Trabalho apresentado do $34^{\circ}$ Congresso Brasileiro de Fitopatologia, 2001.

MOLINA, A., HUNT, M.D., RYALS, J.A Impaired fungicide activity in plants blocked in disease signal transduction. The Plant Cell, Rockville, v.10, n.10, p.1903-1914, 1998.

RIZZO, A.A.N., FERREIRA, M.R., BRAZ, L.T. Ação de acybenzolar-S-methyl (BTH) isolado e em combinação com fungicidas no controle do cancro da haste em melão rendilhado. Horticultura Brasileira, Brasília, v.21, n. 2, p.238-240, 2003.

ROWE, C.R. Potato Health Management. American Phytopathological Society, 1993, 178 p RYALS, J.A., NEUENSCHWANDER, U.H.; WILLITS, M.G.; MOLINA, A., STEINER, H,Y; HUNT, M.D. Systemic acquired resistance. The Plant Cell, Rockville, V.8., n.10, p.1809-1819, 1996.

SIGRIEST, J., GLENEWINCKEL, D., KOLLE, C., SCHMIDTKE, M. Chemically induced resistance in green bean against bacterial and fungal pathogens. Zeitschrift für. Pflanzenkrankheiten. Pflanzenschutz, v.104, n.4, p.599-610, 1997.

SILVA, L.H.C.P., RESENDE, M.L.V., SOUZA, R.M., CAMPOS, J.R. Efeito do indutor de resistência acibenzolar-S-methyl na proteção contra Xanthomonas vesicatoria, Oidium lycopersici e Septoria lycopersici em tomateiro. Summa Phytopathologica, Botucatu, v.29, n.3, p.244-248, 2003.

STICHER, L., MAUCH-MANI, B., MÉTRAUX, J.P. Systemic acquired resistance. Annual Review of Phytopathology, Palo Alto, v.35, p.235-270, 1997. TÖFOLI, G.J., DOMINGUES, R.J., GARCIA JR, O. Desempenho de fungicidas no controle da requeima (Phytophthora infestans) da batata. Fitopatologia Brasileira. Brasília, v.24, 1999. Suplemento. Trabalho apresentado no $32^{\circ}$ Congresso Brasileiro de Fitopatologia, 1999. 
TÖFOLI, G.J., DOMINGUES, R.J., GARCIA JR, O. Controle químico da requeima na cultura da batata. Summa Phytopathologica, Jaboticabal, SP, v.27, 2001. Suplemento. Trabalho apresentado no $24^{0}$ Congresso Paulista de Fitopatologia, 2001. TÖFOLI, J.G. Ação de acibenzolar-S-methyl e fungicidas no controle da pinta preta do tomateiro. Botucatu, 2002, 143 f. (Tese mestrado) - FCA, UNESP, Botucatu.
WEI, G., KOEPPLER, J.W., TUZUN, S. Induction of sistemic resistance of cucumber to Colletotrichum orbiculare by select strains of plant growth promoting rizobacteria. Phytopathology, v.81, n.6, p.1508-1512, 1991.

WHITE, R.F. Acetylsalicylic acid (Aspirin) induces resistance to tobacco mosaic virus in tobacco. Virology, v.99, p.410-412, 1979.
YAMAGUCHI, I. Activators for sistemic acquired resistance. In: HUTSON, D., MYAMAMOTO, J. (Eds.) Fungicidal Activity. New York: Wiley, 1998. p.193-21.

ZAMBOLIM, L.; VALE, F.X.R.; COSTA, H. Doenças causadas por fungos em batata. In: ZAMBOLIM, L.; VALE, F.X., COSTA, H. (Eds.) Controle de doenças de plantas - Hortaliças. Viçosa, 2000. p.173-208. 